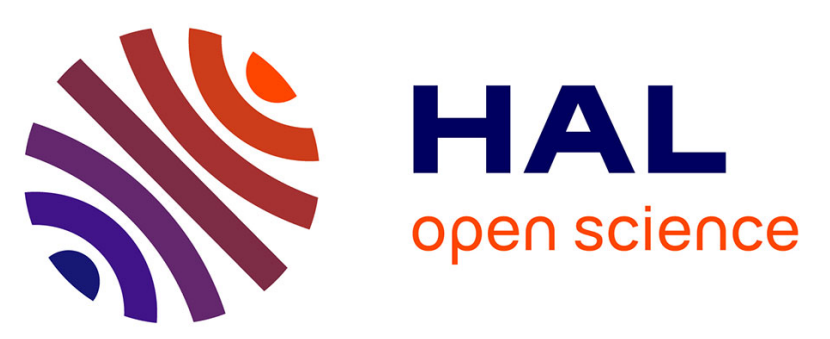

\title{
A study of wake effects on the drag of the Ahmed's squareback model at the industrial scale
}

Mathieu Grandemange, Olivier Cadot, Courbois, A., Vincent Herbert, Denis Ricot, Tony Ruiz, Rémi Vigneron

\section{- To cite this version:}

Mathieu Grandemange, Olivier Cadot, Courbois, A., Vincent Herbert, Denis Ricot, et al.. A study of wake effects on the drag of the Ahmed's squareback model at the industrial scale. Journal of Wind Engineering and Industrial Aerodynamics, 2015, 145, pp.282-291. hal-01205907

\section{HAL Id: hal-01205907 \\ https://hal-ensta-paris.archives-ouvertes.fr/hal-01205907}

Submitted on 29 Sep 2015

HAL is a multi-disciplinary open access archive for the deposit and dissemination of scientific research documents, whether they are published or not. The documents may come from teaching and research institutions in France or abroad, or from public or private research centers.
L'archive ouverte pluridisciplinaire HAL, est destinée au dépôt et à la diffusion de documents scientifiques de niveau recherche, publiés ou non, émanant des établissements d'enseignement et de recherche français ou étrangers, des laboratoires publics ou privés. 


\title{
A study of wake effects on the drag of the Ahmed's squareback model at the industrial scale
}

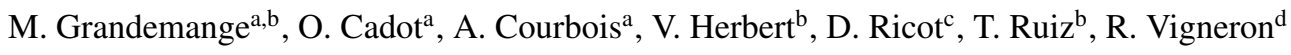 \\ ${ }^{a}$ ENSTA ParisTech, Chemin de la Huniere, 91120 Palaiseau \\ ${ }^{b}$ PSA Peugeot Citron, Route de Gisy, 78140 Vlizy-Villacoublay \\ ${ }^{c}$ Renault SAS, 13/15 Quai Alphonse le Gallo, Boulogne-Billancourt \\ ${ }^{d}$ GIE S2A, 2 Avenue Volta, 78180 Montigny-le-Bretonneux
}

\begin{abstract}
Experiments are performed at industrial scales over the Ahmed geometry, i.e. at a Reynolds number of $R e=2.5 \times 10^{6}$ based on the height of the body. The shape of the squareback geometry is first optimised to make an initial substantial drag reduction. The separated flow at the trailing edge is orientated by introducing chamfers at the top and bottom edges. A parametric study based on both chamfered angles leads to an optimized Ahmed geometry having a drag 5.8\% lower than the reference squareback model. It is evidenced that this optimized geometry produces 4 intense longitudinal vortices that still contribute significantly to the drag. The effect of a sideslip yaw angle is studied. As expected, it is found that the drag increases with an increase in the yaw angle, but surprisingly the drag remains constant for yaw angles within the interval $\pm 0.5^{\circ}$ for which the side force displays very large fluctuations. This plateau is explained by recent observation of the bi-stable properties of the squareback Ahmed body (Grandemange, Gohlke \& Cadot, Physical Review E 86, 2012). The suppression of the bi-stable behavior using a passive control technique is associated with an additional drag reduction of $1.6 \%$.
\end{abstract}

Keywords: Aerodynamics, separation, bi-stability, control, optimisation, induced drag

\section{Introduction}

Car aerodynamics, and more generally ground vehicle aerodynamics is of major interest for future energy saving developments. Due to severe constraints, such as functional shapes, security, and comfort, vehicles have a high aerodynamics drag [1,2]. They belong to the so called bluff bodies associated with strong flow separation and unsteady wake. There are therefore very good reasons to address this problem fundamentally. The work of Ahmed et al. [3] describes the effect of the afterbody shape of a simplified road vehicle on the flow topology and on the drag. In addition to the large recirculating region of low pressure due to the full flow separation at the base, their work proves the critical influence of the slant angle of the rear window. A drag reduction of $8 \%$ compared to the squareback configuration $\left(0^{\circ}\right.$ case $)$ is obtained for a slant inclination of $12.5^{\circ}$. Beyond this optimal value the drag increases continuously and the worst case (slant angle close to $30^{\circ}$ ) is associated with a $50 \%$ increase in drag compared to the squareback geometry. Over a critical angle close to $30^{\circ}$, the drag decreases abruptly to recover values close to the $0^{\circ}$ configuration. In terms of flow topologies, the blunt afterbody is responsible for a massive separation on the base. For slant angles below $30^{\circ}$, the flow remains attached on the rear window 
(at least partially) and a pair of counter-rotating vortices develops from the sides of the slanted face $[4,5,6,7]$ : the intensity of these streamwise vortices gradually grows as the slant angle increases and consequently the drag rises. Above $30^{\circ}$, the flow separates upstream of the rear window and a massive recirculation region without energetic streamwise vortices, similar to the $0^{\circ}$ case, is established. The flow properties of the squareback geometry are investigated both numerically and experimentally in $[8,9,10,11,12]$. The geometry used in the experiments of Ahmed et al. [3] is often used to explore drag reduction strategies strived for industry. Thus, literature reports the use of various different control devices. Much work has been devoted to the $25^{\circ}$ slant angle configuration; significant drag reductions are achieved through passive or active control strategies such as splitter plates [13], flaps [14, 15], boundary layer streaks [16] or even pulsed jets [17]. Diverse strategies equally provide interesting base pressure recovery in the squareback case: splitter plates [18], porous devices [19] or active control [20, 21, 22].

The experiments of Littlewood \& Passmore [23] depict the effect of a small chamfer at the upper trailing edge of a squareback geometry. The geometry of the after body is similar to the squareback Ahmed configuration. Despite the small size of the chamfer in the streamwise direction (only $15 \%$ of the base height), an optimal drag reduction of $4.4 \%$ is obtained for a chamfer angle of $12^{\circ}$. The parabolic-like dependence between the chamfer angle and the drag is reminiscent of the results of Ahmed et al. [3] for moderate slant angles where an optimal $8 \%$ drag reduction is obtained for an angle of $12.5^{\circ}$. Thus, the significant drag reduction obtained by Littlewood \& Passmore [23] with a small chamfer highlights the high impact on force of the flow orientation at the trailing edge. Similarly, Grandemange et al. [24] studied the effect of the angle of small flaps (or spoilers) located at the top and the bottom of the squareback model trailing edge. Their parametric study led to an optimized configuration, with an angle $\Phi_{T}=9.2^{\circ}$ for the top flap and $\Phi_{B}=-7.4^{\circ}$ for the bottom flap. The drag reduction mechanism was explained by a simple two dimensional effect of wake thinning, hence reducing the bluffness of the body [25]. More importantly, the presence of the optimum was related to a drag penalization because of the presence of longitudinal vortices associated with 3D separation at the salient lateral edges of the flaps. This approach using chamfered shapes is particularly interesting since it corresponds to the method applied by the car manufacturers on real vehicles to limit the drag. Indeed, the angles of the spoiler and of the diffuser (when one is present) are set empirically to reach the optimum drag point given the vehicle parameters (global shape, ground clearance...), the characteristic length of these devices remaining small compared to the vehicle size.

Recently, Grandemange et al. [11,12] found that the Ahmed body presents bi-stable wake positions related to a reflectional symmetry breaking in the flow solution. This bi-stability creates large cross flow force fluctuations and probably additional drag. The existence of bi-stability is not only a fact of the Ahmed body, it is related to the aspect ratio of the rectangular blunt trailing edge of the 3D body together with the ground clearance [26]. For instance, it has has been observed for a double backward facing step geometry [27]. In car aerodynamics, Lawson et al. [28] observed such behaviour for a realistic car model at 1/4 scale. For the Ahmed body, the bi-stability was found particularly sensitive to steady disturbances when placed in the middle of the recirculation bubble. A vertical cylinder, having the body height and only $5.6 \%$ the body width in diameter is able to suppress the bi-stability together with producing a drag reduction estimated to be between $5 \%$ and $8 \%$ [29].

The goal of this article is to investigate these new flow understandings and their corresponding drag improvements at the industrial scale while considering two main questions:

(i) can the substantial drag contribution of the longitudinal vortices near the lateral edges of the chamfers be evidenced? 
(ii) can the substantial drag contribution due to the wake bi-stability be evidenced ?

The simplified Ahmed body is still employed, but in real flow condition with road effect and rotating wheels.

The article is organized as follows. The experimental set-up is first described in section 2 . The parametric study of small chamfers at the top and bottom of the trailing edge in section 3.1 leads to an optimal drag configuration. This optimal drag model is then used as a reference to test drag reduction methods in the next two sections. In section 3.2, the bi-stable behavior of the wake is evidenced through sideslips measurements and then controlled to successfully reduce the drag. In section 3.3, the longitudinal vortices are suppressed by introducing chamfers on the lateral sides and proves their important drag contribution to the optimal configuration with top and bottom chamfers. Finally, the concluding remarks are presented in Sec. 4.

\section{Experimental setup}

Figure 1 illustrates the actual set-up for the experiments. It is similar to the squareback Ahmed gemetry used in [3], but is 4 times larger, has a chamfered appendix (shown at (ii) in the diagram) and has a top rear edge chamfered at an angle $\Phi_{T}>0$ (shown at (i) in the diagram). Also, the original cylindrical supports are replaced by proper wheels, and the distance to the floor is larger. In [3], the separating distance is 0.186 the body height while it is 0.165 in the present study. The body is tested in the full scale wind-tunnel of GIE S2A at Montigny-Le-Bretonneux especially designed for automotive aerodynamics. A full description of the facility may be found in [30]. Briefly, the test section is a $3 / 4$ open jet with a cross-section of $24 \mathrm{~m}^{2}$. The body is placed on a large turntable to reproduce sideslip situations with a yaw angle $\beta$ as defined in figure $1(b)$. A wide belt is entrained at the wind velocity between the rotating wheels to introduce a real road effect on the underbody flow.

The measurements systems are provided by the GIE S2A. The model is fixed by four thin supports (two downstream the front wheels, and two upstream the rear wheels) on a 6 components balance which provides both the mean value and the standard deviation of each component of the force, say $F_{i}$ and $\operatorname{Std}\left(F_{i}\right)$ with $i \in\{x, y, z\}$ denotes respectively, the drag, the side and the lift force component. These quantities are obtained from recordings at a sampling frequency of $10 \mathrm{~Hz}$ during $40 \mathrm{~s}$ when a statistically steady state is reached; part of the fluctuations are ascribed to measurement uncertainty but the values of $\operatorname{Std}\left(F_{i}\right)$ still give qualitative information on the steadiness of the forces. Parietal static pressure are obtained on the geometry at 47 locations: 35 taps are placed on the base as presented in figure 1(c), the 12 others are distributed in the middle of the top, bottom and lateral faces at $x^{*}=-0.22,-0.43$ and -1.30 . When additional information is needed, other pressure sensors are set at some precise locations. Standard pressure measurements are obtained from signal sampled at $2.5 \mathrm{~Hz}$, usually averaged over $40 \mathrm{~s}$.

Measurements in the wake use three 18 holes spherical probes mounted on a three-axis traversing system [30]. The probes are "omniprobe" models from Aeroprobe Corporation having diameters of $9.53 \mathrm{~mm}$. The measurement is performed continuously at an acquisition rate of $2.5 \mathrm{~Hz}$ while the traversing system is moving at a constant speed of $75 \mathrm{~mm} \cdot \mathrm{s}^{-1}$. Hence, one measurement point is obtained each $30 \mathrm{~mm}$ that sets the spatial resolution. It gives the mean velocities $U_{x}, U_{y}$ and $U_{z}$ and its gradients after processing as well as the static pressure. The precision of the velocity is given by the manufacturer to be less than $1 \%$ of the measured velocity modulus, and the angular precision to be less than $0.4^{\circ}$. The automatic displacement allows to get these data in planes iso- $x$, iso- $y$ or iso- $z$. 
(a)
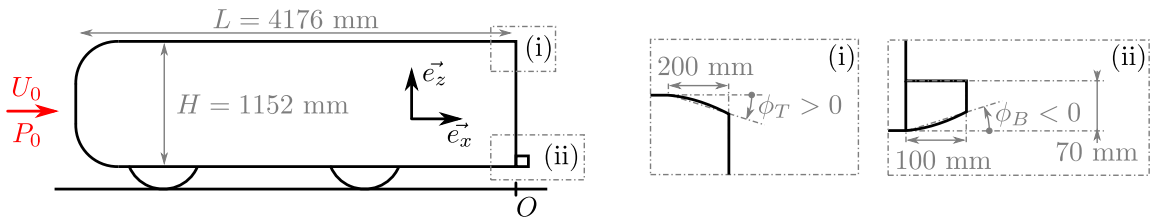

(b)
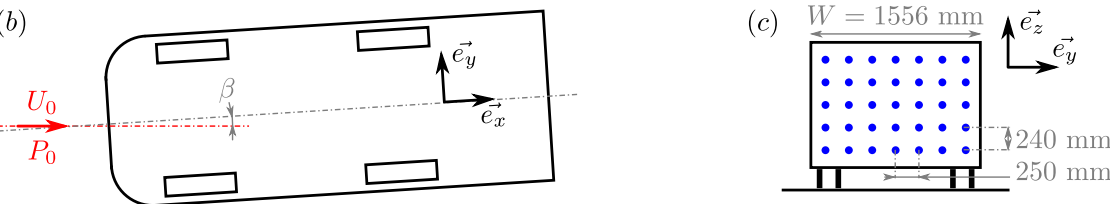

Figure 1: Layout of the squareback Ahmed geometry used for the investigations. Side view in shown at $(a)$, top view at $(b)$ and back view at $(c)$. $O$ sets the origin of the coordinate system. The blue dots locate the pressure taps on the base. For the reference squareback Ahmed geometry (SB) (see table 1), there is no appendix (ii) and $\Phi_{T}=0^{\circ}$ in (i).

For all the tests, the inlet velocity is set at $U_{0}=33.3 \mathrm{~m} \mathrm{~s}^{-1}$. The Reynolds number based on the height $H$ of the geometry (see figure 1a) is $\operatorname{Re}=\frac{U_{0} H}{v}=2.510^{6}$. In the following, $a^{*}$ denotes the non-dimensional value of any quantity $a(x, y, z, t)$ made dimensionless by a combination of the height $H$ and the inlet velocity $U_{0}$. The force and the pressure coefficients are defined as :

$$
C_{i}=\frac{F_{i}}{\frac{1}{2} \rho S U_{0}^{2}} ; C_{p}=\frac{p-p_{0}}{\frac{1}{2} \rho U_{0}^{2}},
$$

with $i$ referring to the force component, $\rho$ is the air density, $S=H \times W$ is the frontal area of the Ahmed body and $p_{0}$ is the free stream pressure. The coefficients are given to be accurate in the absolute range \pm 0.002 .

\section{Results}

The force coefficients of the squareback Ahmed model are displayed in table 1. The drag coefficient is 0.309 which is larger than the value of 0.250 obtained in [3] despite the fact that the Reynolds numbers of our study is only twice the Reynolds number in [3]. The larger drag is then ascribed to the differences in the ground clearances, the rotating wheels and the road effect. The lift coefficient $C_{z}$ is slightly negative. The fluctuation of the side force coefficient $S t d\left(C_{y}\right)$ is very large compared to the values of the fluctuations in lift and drag. It is related to the wake bi-stability $[11,12]$ as it will be shown and discussed in section 3.2.

\subsection{Drag optimisation by flow orientation at the top and the bottom of the base}

This section studies at industrial scales the drag dependence on the flow orientation at the base presented in [24]. Our objective here is to obtain an optimized Ahmed model with substantial drag reduction compared to that of the squareback geometry by chamfering the trailing edge. The top edge of the squareback model is chamfered with positive or negative angles as indicated in the dashed box (i) in figure 1. For practical reasons, we had to introduce an appendix at the bottom edge of the squareback model in order to orientate the flow as indicated in the dashed box (ii) in figure 1. The geometry of the chamfers is a circle arc with an horizontal tangent at the 
Table 1: Mean and standard deviation of the force coefficients for the squareback model (SB), and 3 configurations of wake control: optimized model $(\mathrm{O})$ with top and bottom chamfers $\left(\Phi_{T}=7.5^{\circ}, \Phi_{B}=-5^{\circ}\right)$, the optimized model disturbed with vertical cylinder of different diameter sizes placed in the centre of the recirculating region (O-D1 and O-D2). The SB model is used as the reference to compute the drag reductions (DR).

\begin{tabular}{l|ccccccc} 
Configuration & $C_{x}$ & $D R$ & $C_{y}$ & $C_{z}$ & $S t d\left(C_{x}\right)$ & $S t d\left(C_{y}\right)$ & $S t d\left(C_{z}\right)$ \\
\hline SB : squareback (ref.) & 0,309 & - & 0,001 & $-0,047$ & 0.004 & 0.015 & 0.006 \\
O : optimized & 0.291 & $-5.8 \%$ & -0.003 & -0.014 & 0.003 & 0.012 & 0.005 \\
O-D1 : disturbance 5cm & 0.286 & $-7.4 \%$ & 0.002 & -0.009 & 0.004 & 0.007 & 0.005 \\
O-D2 : disturbance 2cm & 0.291 & $-5.8 \%$ & 0.004 & -0.014 & 0.003 & 0.012 & 0.005
\end{tabular}

(a)

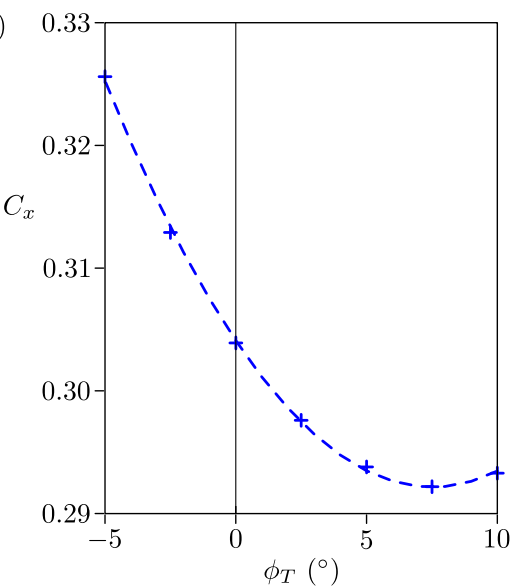

(b)

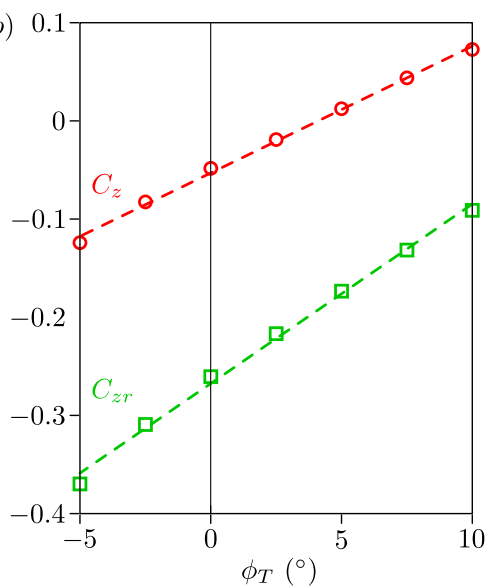

Figure 2: Drag (a) and lift $(b)$ for different angles $\phi_{T}$ with $\phi_{B}=0^{\circ}$; the dashed lines are $1^{\text {st }}$ and $2^{\text {nd }}$ order polynomial fits. 


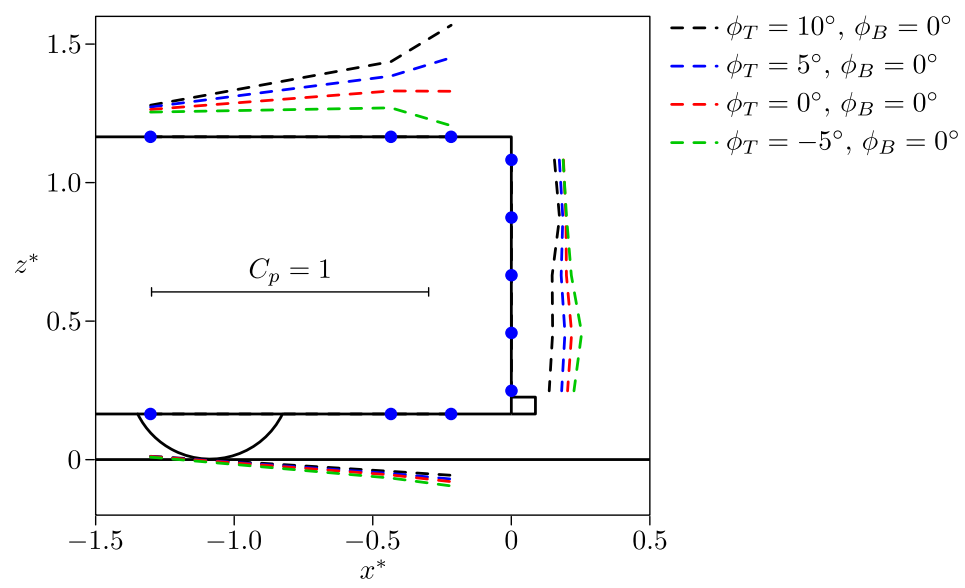

Figure 3: Pressure coefficient levels on the afterbody in the plane of symmetry for different angles $\phi_{T}$ with $\phi_{B}=0^{\circ}$. The blue dots locate the pressure taps. The pressure coefficient is always negative and quantified by the distance from the surface (the length for $C_{p}=1$ is indicated).

upstream edge. The angles $\Phi_{T}$ and $\Phi_{B}$ are defined by the line passing through the upstream and the downstream edge of the chamfers as depicted in figure 1(a).

As a first step, the bottom angle is set to $\phi_{B}=0^{\circ}$ and the effects of the top angle are considered. The forces as functions of $\phi_{T}$ are presented in figure 2. They confirm the trends and amplitudes obtained in [24] as the drag is measured as a $2^{\text {nd }}$ order polynomial fit while the lift presents an affine relationship. Besides, the rear lift ${ }^{1}$, denoted $C_{z r}$, is equally an affine function of the top slant angle corroborating the fact that the lift evolutions are mostly ascribed to pressure modification on the afterbody. However, one can see a slight difference of slope between $C_{z}$ and $C_{z r}$ in figure 2 ; this indicates that there are still some small variations of the lift on the front axle.

The pressure distribution on the afterbody in the plane $y^{*}=0$ are presented in figure 3 for different top angles $\phi_{T}$. As in [24], the effect is clear on the pressure distribution on the top face. The curvature of the geometry is transferred to the potential flow. This results in increases or decreases in pressure depending on the sign of $\phi_{T}$, just upstream of the slanted surface for $x^{*}>-1.0$. The effect is also clear on the base pressure, especially on the gradient in the $z$ direction. Finally, one can observe some modifications of the pressure on the lower face evidencing the coupling effects of the top and bottom angles.

The quadratic dependence between the drag and $\phi_{T}$ combined with the affine dependence between the lift and $\phi_{T}$ leads to a relationship between the drag and the lift that can be expressed by

$$
C_{x}-C_{x 0}=\alpha\left(C_{z}-C_{z 0}\right)^{2} .
$$

These relationships are presented in figure 4(a) for different bottom angles $\phi_{B}$. As in the experiments in [24], all the parabole are self-similar with a parameter $\alpha=1.27$ independent of $\phi_{B}$ at first order (see figure $4 b$ ). It is remarkable to see that the value of $\alpha$ is identical to the one

\footnotetext{
${ }^{1}$ The rear lift is the part of the lift measured on the rear axle of the geometry.
} 
$(a)$

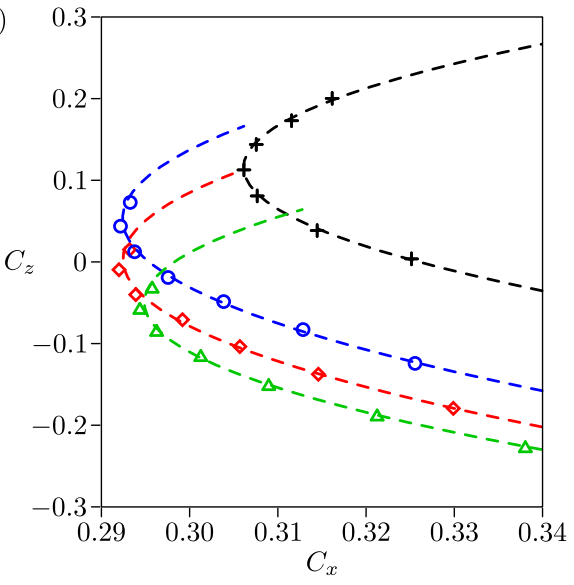

(b)

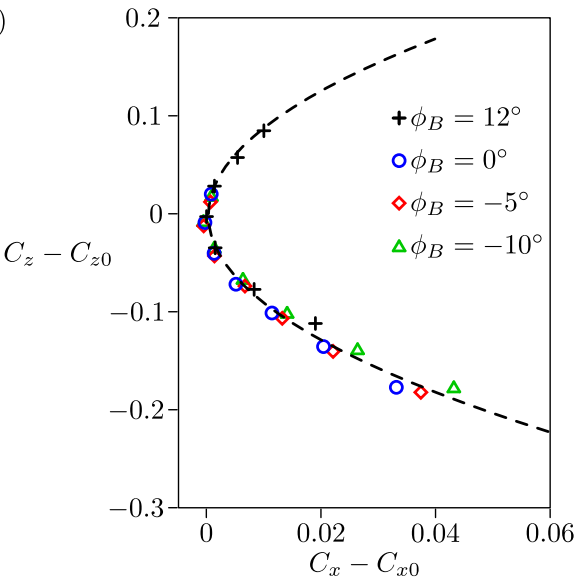

Figure 4: Drag vs. lift $(a)$ and data centered on their respective extremum $(b)$ as a parametric function of the top slant angle $\phi_{T}$ for different $\phi_{B}:+, \phi_{B}=12^{\circ} ; \bigcirc, \phi_{B}=0^{\circ} ; \diamond, \phi_{B}=-5^{\circ} ; \triangle, \phi_{B}=-10^{\circ} ;--, 2^{\text {nd }}$ order polynomial fits.

obtained in [24] which proves that the force evolutions are of similar amplitude. Moreover, this is consistent with the geometrical interpretation of $\alpha$ in the theory of induced drag.

These variations of force intensities are associated with important modifications of the flow topology. The flows obtained with the two extreme lift configurations are compared to the flow obtained for $\phi_{T}=\phi_{B}=0^{\circ}$ in figures 5, 6 and 7. In the plane $y^{*}=0$, the results plotted in figure 5 show that the top - bottom equilibrium of the flow is strongly modified. The recirculation bubble is almost symmetric for $\phi_{T}=\phi_{B}=0^{\circ}$ while it is significantly asymmetric in the extreme cases. So, the different couples of angles considered in these experiments should explore all the wake orientations between figures 5(a) and 5(c).

The three-dimensional effects play a first order role in the drag. Figure 6 presents the streamwise vorticity in the plane $x^{*}=0.43$ for the three different configurations. These maps confirm the formation of streamwise vortices downstream of the corners of the base. The dashed lines locate the contour $U_{x}{ }^{*}=0.5$ to discriminate the energetic vortices in the high velocity flow from the chaotic vorticity obtained in the recirculation bubble, mostly due to measurement uncertainty. Besides, for the case $\phi_{T}=\phi_{B}=0^{\circ}$ in figure $6(b)$, there are clear vortices downstream of the underbody in spite of the absence of bottom inclination; they may be ascribed to the presence of rotating wheels.

Further downstream, at $x^{*}=2.60$, figures $7(a)$ and $7(c)$ evidence that the concentrated vortices merge from each side to form a single pair of large scale counter-rotating vortices for the configurations $\left(\phi_{T}=-5^{\circ}, \phi_{B}=-10^{\circ}\right)$ and $\left(\phi_{T}=10^{\circ}, \phi_{B}=12^{\circ}\right)$. The presence of these vortices and their directions of rotation are consistent with the lift measured at $C_{z}=-0.22$, $C_{z}=-0.05$ and $C_{z}=0.20$ for the configurations $\left(\phi_{T}=-5^{\circ}, \phi_{B}=-10^{\circ}\right),\left(\phi_{T}=0^{\circ}, \phi_{B}=0^{\circ}\right)$ and $\left(\phi_{T}=10^{\circ}, \phi_{B}=12^{\circ}\right)$ respectively.

As a consequence, the general trends and topologies ascribed to the different angles reported in [24] remain valid at higher Reynolds numbers. The optimal drag configuration obtained for $\phi_{T}=7.5^{\circ}$ and $\phi_{B}=-5.0^{\circ}(\mathrm{O}$ model in table 1$)$ with a $5.8 \%$ drag reduction is now considered. The corresponding flow is presented in figure 8 . The velocities in the plane $y^{*}=0$ show that 

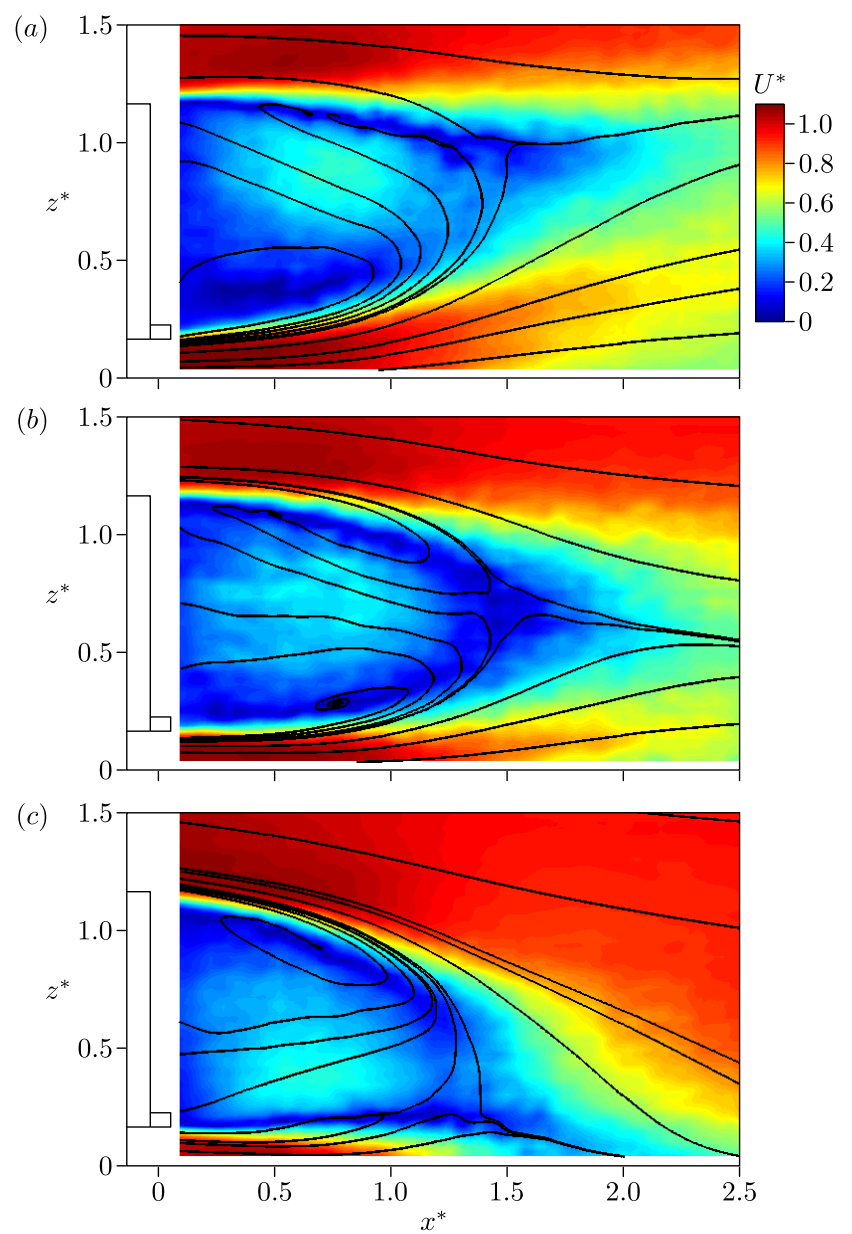

Figure 5: Velocity in the plane $y^{*}=0$ for $\phi_{T}=-5^{\circ}$ and $\phi_{B}=-10^{\circ}(a), \phi_{T}=0^{\circ}$ and $\phi_{B}=0^{\circ}(b), \phi_{T}=10^{\circ}$ and $\phi_{B}=12^{\circ}$ (c). 
(a)

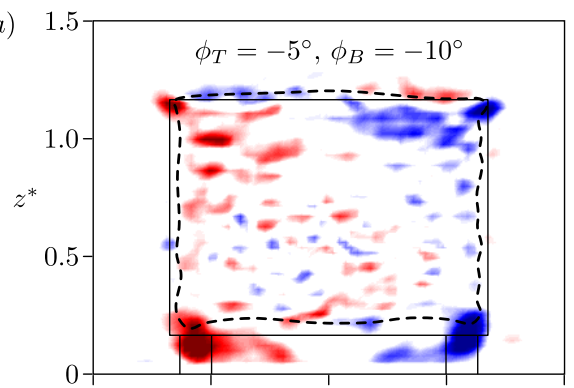

(c)

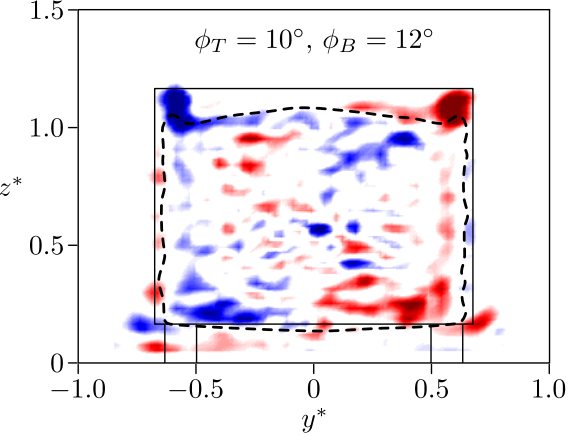

(b)

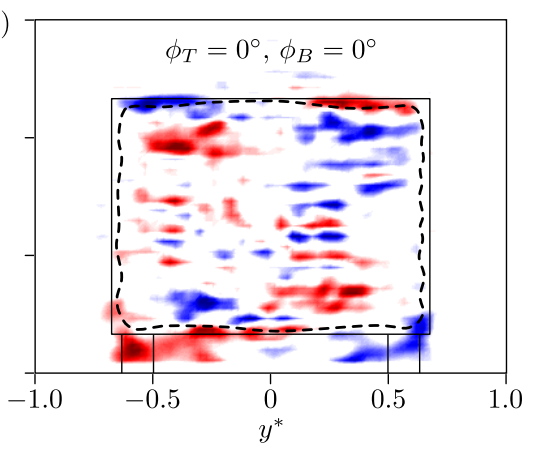

$\Omega_{x}{ }^{*}$

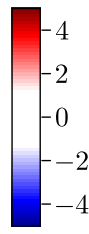

Figure 6: Streamwise vorticity in the plane $x^{*}=0.43$ for $\phi_{T}=-5^{\circ}$ and $\phi_{B}=-10^{\circ}(a), \phi_{T}=0^{\circ}$ and $\phi_{B}=0^{\circ}(b)$, $\phi_{T}=10^{\circ}$ and $\phi_{B}=12^{\circ}(c)$. The dashed line is the contour $U_{x}{ }^{*}=0.5$.

(a)

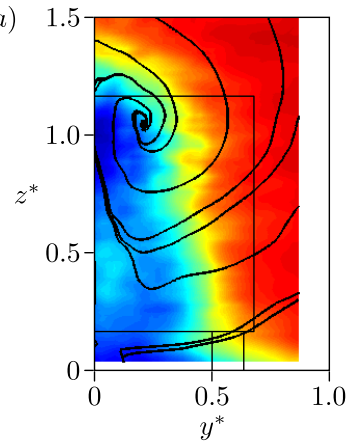

(b)

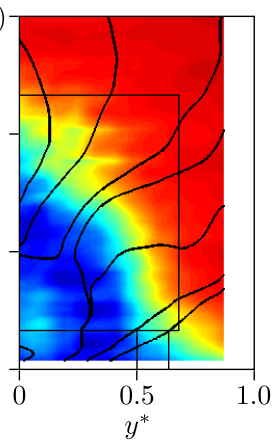

(c)

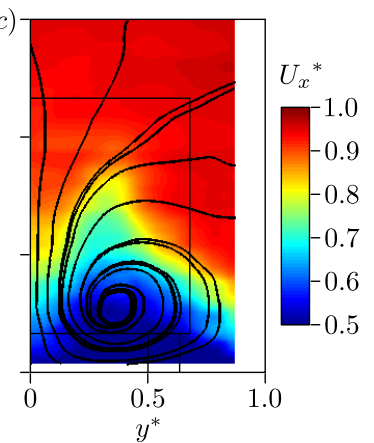

Figure 7: Velocity in the plane $x^{*}=2.60$ for $\phi_{T}=-5^{\circ}$ and $\phi_{B}=-10^{\circ}(a), \phi_{T}=0^{\circ}$ and $\phi_{B}=0^{\circ}(b), \phi_{T}=10^{\circ}$ and $\phi_{B}=12^{\circ}(c)$. 

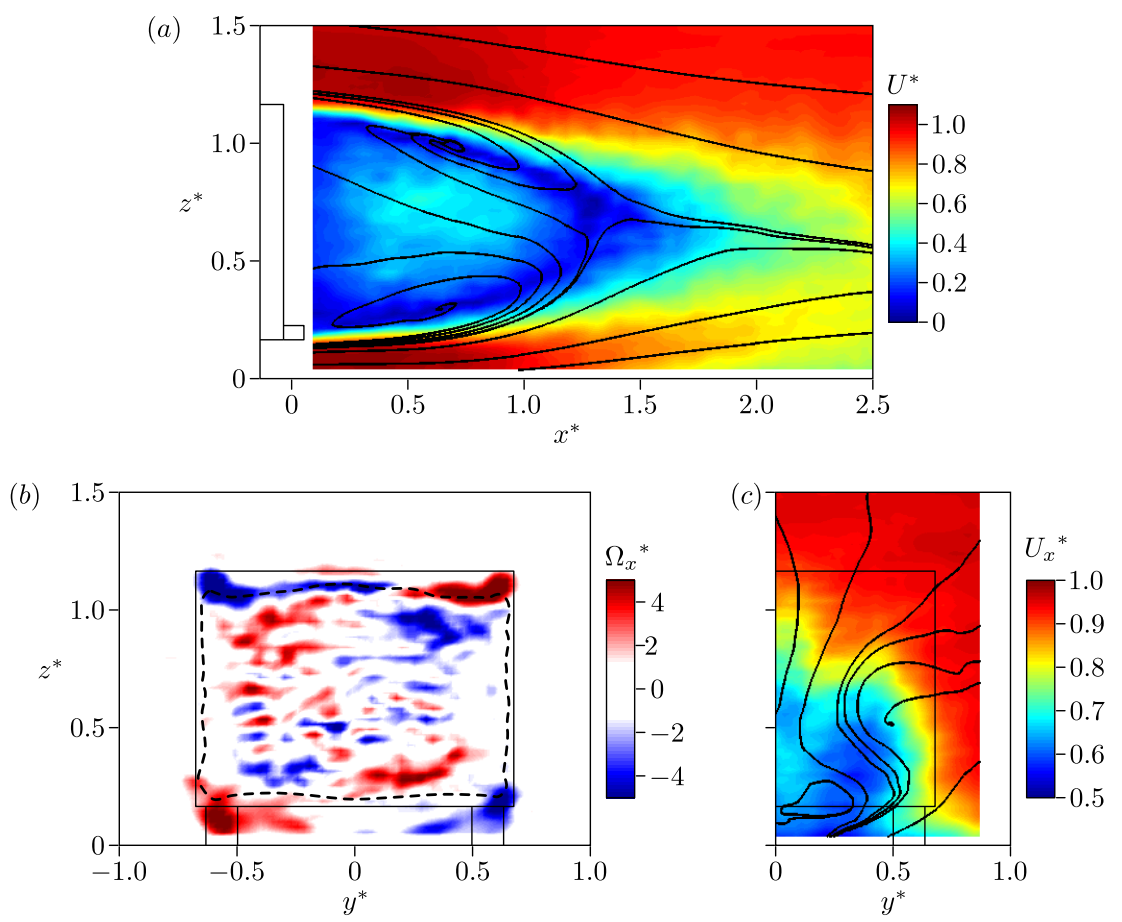

Figure 8: Flow around the optimal configuration $\phi_{T}=7.5^{\circ}$ and $\phi_{B}=-5^{\circ}(\mathrm{O}$ model in table 1): velocity in the plane $y^{*}=0(a)$, streamwise vorticity in the plane $x^{*}=0.43(b)$ and velocity in the plane $x^{*}=2.60(c)$. The dashed line in $(b)$ is the contour $U_{x}{ }^{*}=0.5$.

the flow is slightly asymmetric with a reduced recirculation region in comparison to the case $\phi_{T}=\phi_{B}=0^{\circ}$ (see figure $8 a$ ). The streamwise vorticity in the very near wake is visible in figure $8(b)$; it evidences the presence of counter-rotating vortices downstream of the corners of the flaps. The vortices emitted from the same side of the geometry are very likely to attenuate each other which results in the absence of large-scale vortices further downstream: only residual traces of vortices are visible in the streamlines in figure $8(c)$.

As in the model of drag sources [24] deduced from the drag optimization using orientated flaps at the top and bottom trailing edge, the optimized geometry results in a compromise between the reduced bluffness at the rear and the formation of streamwise vorticity at both ends of the chamfers. This can be seen by comparing the natural case in figure 6(b) to the optimized geometry in figure $8(\mathrm{~b})$. The height of the separated region depicted with the dashed line is smaller for the optimized case, but the streamwise vortices are more intense. The idea in [24] is to ascribe a source of drag decrease by the wake thinning, which takes into account two dimensional effects only, and a source of drag increase resulting from the drag induced by the streamwise vortices which takes into account three-dimensional effects. The latter depends on the square of the lift force exerted on the chamfers as for the finite size effect of wings in aeronautics.

To gain more understanding of the flow, control experiments explore both the sensitivity of this optimized configuration, especially regarding a possible bi-stable behavior in section 3.2 and some perspectives to limit the formation of the streamwise vortices due to the chamfered trailing 
(a)

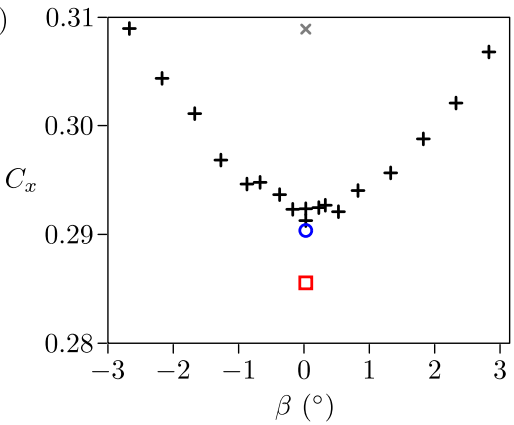

(b)

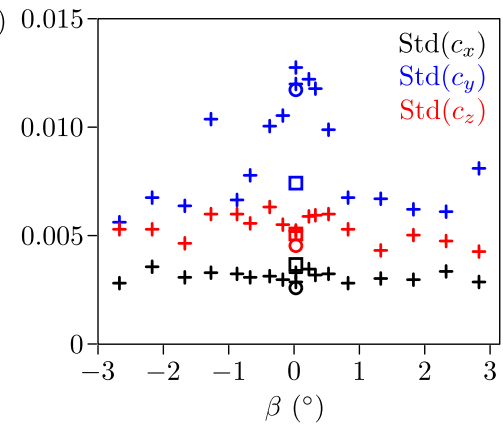

Figure 9: Drag $(a)$ and fluctuations of forces $(b)$ as a function of a slight yaw angle $\beta: \times$, reference SB model;,$+ \mathrm{O}$ model; $\square$, OD-1 model (disturbing square cylinder of $5 \mathrm{~cm}$ side); $\bigcirc$, OD-1 model (disturbing circular cylinder of $2 \mathrm{~cm}$ diameter) The disturbing cylinders are placed at $x_{C}{ }^{*}=0.87$ and $y_{C}{ }^{*}=0$. See table 1 for the different models configuration and force coefficient measurements.

edge in section 3.3 .

\subsection{Sideslip effect, wake bi-stability and its control}

One of the capabilities of this wind tunnel is the possibility to set a slight and accurate yaw angle; this provides a quantitative parameter of the setup asymmetry. The force measurements allow a detailed description of the effects of a small yaw angle. The drag and the fluctuations of forces as functions of $\beta$ are plotted in figure 9. The drag evolution can be decomposed into two regions. There is an affine drag increase as the modulus of $\beta$ increases for $|\beta|>0.5^{\circ}$ whereas the drag saturates (given the accuracy of the measurements) on a plateau in the range $|\beta|<0.5^{\circ}$. Note that the linear trend is equally reported in the experiments of [31] and is likely to extend up to $|\beta| \approx 15^{\circ}$. The fluctuations of drag and lift are independent of the yaw angle but the side force presents strong fluctuations for $|\beta|<0.5^{\circ}$ in correspondence to the region of the saturated drag value. These fluctuations are governed by the coexistence of two mirror states (bi-stability) in the wake as evidenced in [12]. The pressure distributions on the base for $\beta=-0.7^{\circ}, 0^{\circ}$ and $0.8^{\circ}$ are plotted in figure 10. Both extreme angles present a predominance of one of the two asymmetric states, using the same terminology as in [12], the state \#N is predominant for the negative yaw angle in figure 10(a) and state \#P is predominant for the positive yaw angle in figure 10(c). For the nil yaw angle, the residual asymmetry in figure 10(b) indicates that both states are not equiprobable, but with a small predominance of state \#P. Hence, the asymmetry introduced by a slight yaw angle $\beta$ modifies the states proportion \#P and \#N. For yaw angles such that $|\beta|>1^{\circ}$, the wake is locked in one state which explains the low and constant side force fluctuation. This is similar to the wake manipulation in [29] using a small control cylinder that is able to change the proportion of each state \#P and \#N keeping a constant drag of the Ahmed body. In the present case, the drag is also insensitive to the states proportion because of the presence of the plateau in the range of small yaw angles.

Now, as in the laboratory experiments of [29], the drag associated with the bi-stability may be quantified using a vertical disturbance. They showed that a vertical cylinder placed in the middle of the recirculation region suppresses the bi-stability which enables a drag reduction to be achieved. To confirm the sensitivity of the asymmetric states to such a disturbance, a similar experiment is performed here at the industrial scale. A vertical control cylinder is placed at 
(a)

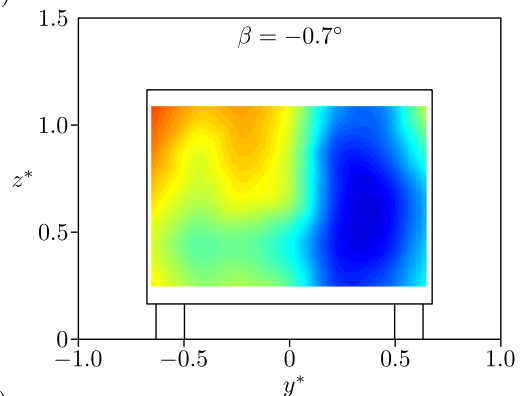

(c)

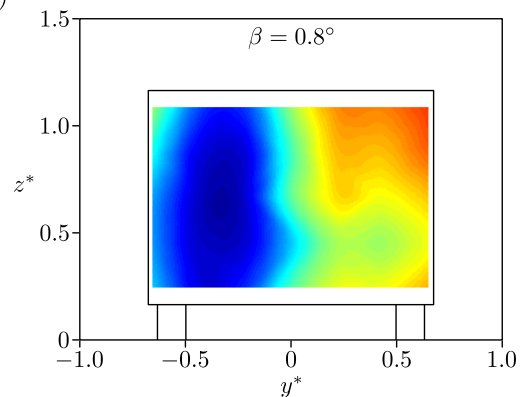

(b)

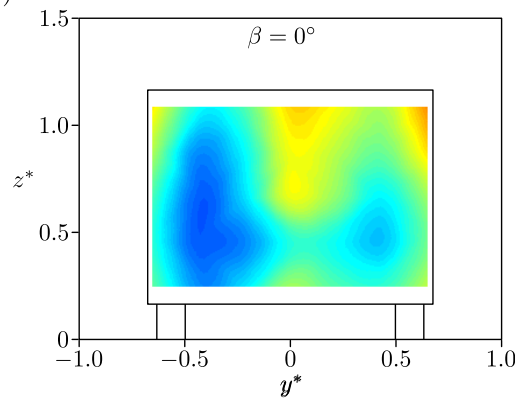

$C_{p}$

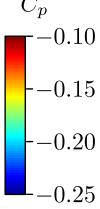

Figure 10: Base pressure distribution for different sideslip yaw angles of the $\mathrm{O}$ model: $\beta=-0.7^{\circ}(a), \beta=0^{\circ}(b)$ and $\beta=0.8^{\circ}(c)$.

$x_{C}{ }^{*}=0.87$ and $y_{C}{ }^{*}=0$; it has a square cross section with a length matching the height of the body and a side of $50 \mathrm{~mm}$, i.e. $4.3 \%$ of the body height. Practically, it is supported by a rod of $20 \mathrm{~mm}$ in diameter, fixed on the wind tunnel floor. As in [29], the drag of the control cylinder is not measured. The control cylinder position is at the end of the sensitive region obtained in [29]. The associated force measurements are given in table 1 and plotted in figure 9 with the square symbols for $\beta=0^{\circ}$. The drag is slightly decreased, by approximately $1.7 \%$ compared to the optimized model, but a significant effect is obtained on the fluctuations of the side force. The levels of $\operatorname{Std}\left(C_{y}\right)$ are reduced from 0.015 to 0.007 , the latter value being close to the expected value for a stable configuration. A smaller but significant effect is reported using a smaller control cylinder of diameter $20 \mathrm{~mm}$, i.e. $1.7 \%$ of the body height, even if this disturbance is much less efficient in stabilizing the wake and the drag reduction hardly measurable (see figure 9).

One can note that the use of a disturbance leads to the minimum drag point presumed from the linear dependencies on the yaw angle for $|\beta|>1.0^{\circ}$. However, the drag reductions obtained in these experiments after suppression of the bi-stability are clearly smaller than the 5 to $8 \%$ expected from the results of [29]. This could be ascribed to a non-optimal position of the disturbance $^{2}$. However, we do not rule out that the disturbance may also produce other effects, not yet identified, independent from the bi-stability suppression and sensitive to the Reynolds number. Further analysis is needed for definite conclusions. 
Table 2: Mean and standard deviation of the force coefficients for the squareback model extended with a straight cavity (C), and 3 configurations of flow orientation combinations at the trailing edge. The C-TB configuration has top and bottom chamfers $\left(\Phi_{T}=10^{\circ}, \Phi_{B}=-10^{\circ}\right)$, C-LR has left and right chamfers $\left(\Phi_{L}=10^{\circ}, \Phi_{R}=-10^{\circ}\right)$ and C-TBLR is combining the two previous configurations with chamfers at all edges.

\begin{tabular}{l|ccccccc} 
Configuration & $C_{x}$ & $D R$ & $C_{y}$ & $C_{z}$ & $S t d\left(C_{x}\right)$ & $S t d\left(C_{y}\right)$ & $S t d\left(C_{z}\right)$ \\
\hline C : straight cavity (ref.) & 0.298 & - & 0.016 & -0.040 & 0.003 & 0.008 & 0.006 \\
C-TB : top \& bot & 0.298 & $0 \%$ & 0.019 & -0.065 & 0.004 & 0.005 & 0.004 \\
C-LR : left \& right & 0.293 & $-1.7 \%$ & 0.028 & -0.052 & 0.004 & 0.008 & 0.007 \\
C-TBLR : all chamfered & 0.276 & $-7.4 \%$ & 0.035 & -0.069 & 0.003 & 0.007 & 0.008
\end{tabular}

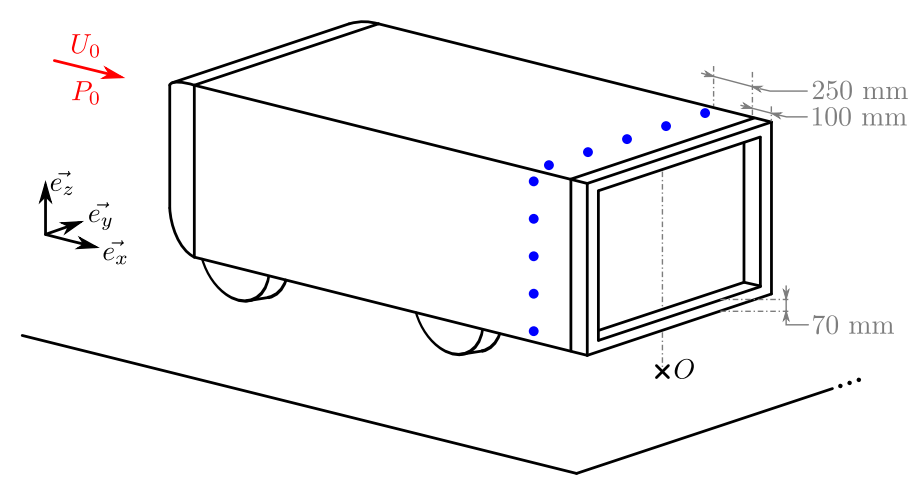

Figure 11: Scheme of the $\mathrm{C}$ model geometry with the cavity at the trailing edge; the blue dots locate the pressure taps added for these experiments. 

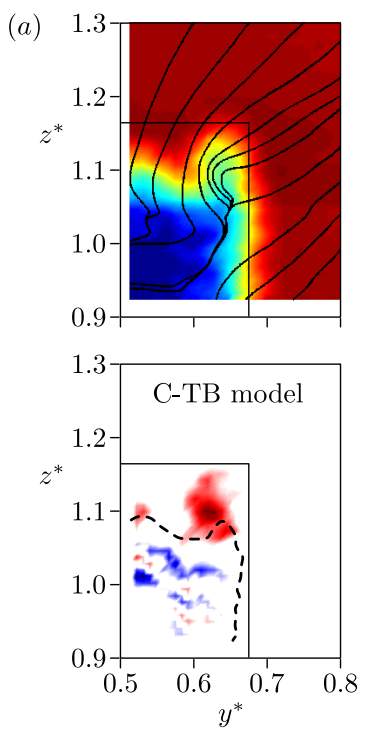
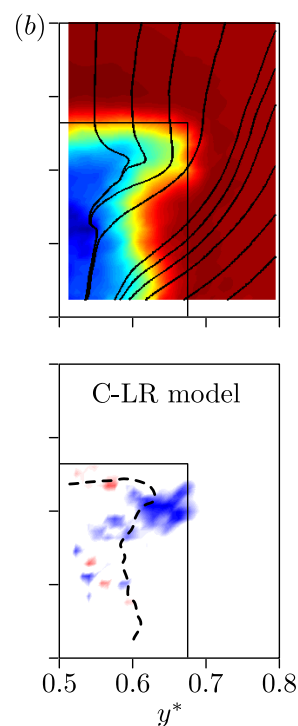
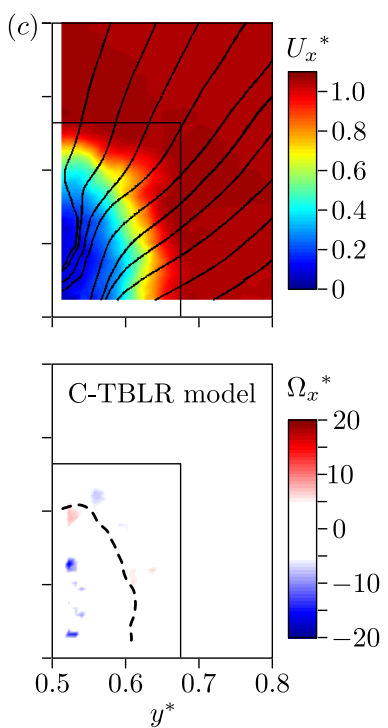

Figure 12: Velocity (top) and streamwise vorticity (bottom) in the plane $x^{*}=0.35$ downstream of the upper corner of the base: C-TB model $\left(\phi_{T}=-\phi_{B}=10^{\circ}\right.$ and $\left.\phi_{L}=\phi_{R}=0^{\circ}\right),(a)$; C-LR model $\left(\phi_{T}=\phi_{B}=0^{\circ}\right.$ and $\left.\phi_{L}=-\phi_{R}=10^{\circ}\right)$, (b); C-TBLR model $\left(\phi_{T}=-\phi_{B}=\phi_{L}=-\phi_{R}=10^{\circ},(c)\right)$. See table 2 for the different models configuration and force coefficient measurements.

\subsection{Flow orientation at the four sides of the base with cavity}

The squareback trailing edge is now slightly extended downstream (by a length of $100 \mathrm{~mm}$ ) as depicted in figure 11. Four configurations are studied, they are listed in table 2.

For the first configuration denoted $\mathrm{C}$, the extension is straight on the four sides. A drag reduction of $3.6 \%$ is obtained with $C_{x}=0.298$ for the $\mathrm{C}$ model against $C_{x}=0.309$ for the squareback Ahmed body (table 1). It is known that deep cavities at the base improve the drag [32], which is particularly employed as a major drag reducer for trucks [33]. The second configuration, called $\mathrm{C}-\mathrm{TB}$, uses the flow orientation at the top and bottom trailing edge. Both the top and the bottom extensions are thus chamfered as in the previous part, but with larger angles, of $\Phi_{T}=-\Phi_{B}=10^{\circ}$, than the optimized Ahmed body. For these larger chamfers, the C-TB geometry has no drag improvement (see table 2) since it is not the optimum. Particularly, we can see the presence of longitudinal vortices in figure 12, that are associated with three dimensional separations produced by global pressure differences between the body faces. The pressure distribution on the sides of the geometry at $x^{*}=-0.22$ presented in figure 13 supplies a measurement of these global pressure levels on each face. We can see in figure 13(a) that the pressure levels on the C-TB model are only affected at the top and bottom faces compared to the $\mathrm{C}$ model. Because of the curvature introduced by the flow orientation at the edges, the pressure level on the top and bottom faces is lower than that on the left and right faces. The pressure differences are responsible for the three dimensional separation and vortex formation around each corner at the base.

${ }^{2}$ The cylinder is placed manually so an exploration of a domain $\left(x_{C}{ }^{*}, y_{C}{ }^{*}\right)$ is long and tedious. 
(a)
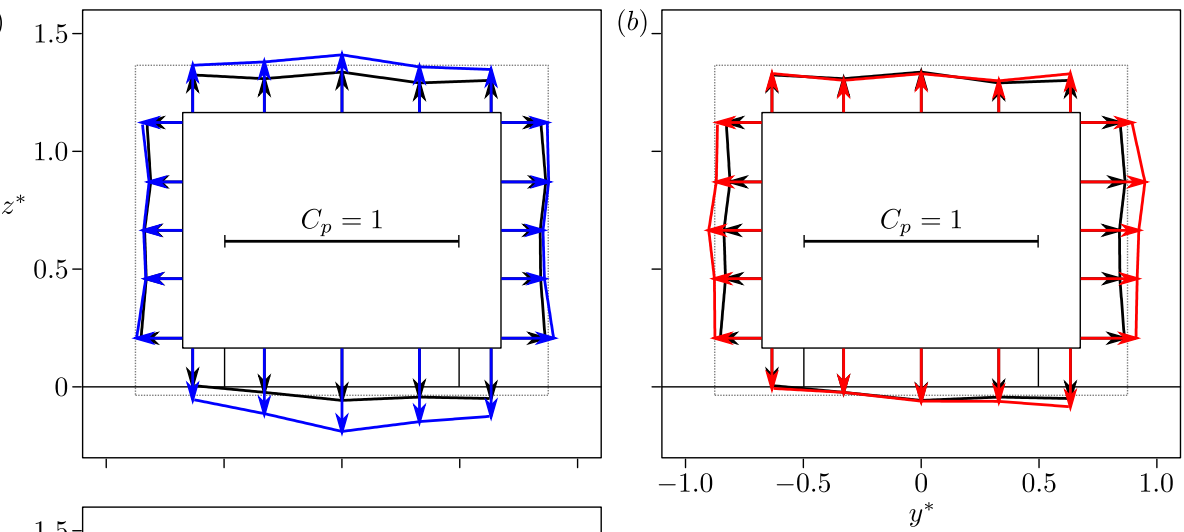

(c) 1.5

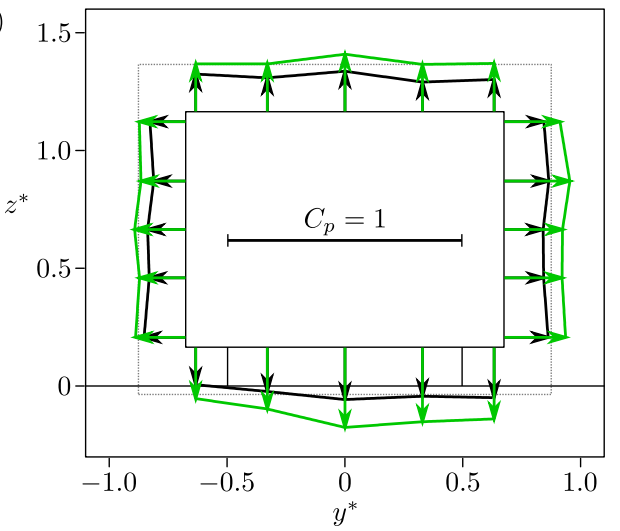

C model

C-TB model

C-LR model

- C-TBLR mode

Figure 13: Levels of pressure coefficients around the geometry at $x^{*}=-0.22$ for the different combinations of flow orientations at the trailing edge. Comparisons between C model and C-TB model (a); C-LR model (b); C-TBLR model (c). See table 2 for the different models configuration and force coefficient measurements. The arrows locate the pressure taps; the pressure coefficient is always negative and quantified by the distance from the surface (the length for $C_{p}=1$ is indicated) and the dotted gray lines locate the contours $C_{p}=-0.2$ 
We turn now to the C-LR model for which only both lateral edges are chamfered with $\Phi_{L}=$ $-\Phi_{R}=10^{\circ}$ (see table 2). In that case, only the pressure levels on the left and right faces are significantly decreased compared to the $C$ model (figure 13b). It results in three dimensional separation producing longitudinal vortices at each corners but with opposite vorticity to that of the C-TB model (see figure 12b). A drag reduction of $1.7 \%$ is obtained for the C-LR model.

The very interesting result is for the flow orientation at all the edges $\Phi_{T}=-\Phi_{B}=10^{\circ}$ and $\Phi_{L}=-\Phi_{R}=10^{\circ}$, better known as the "boat tail" geometry. The C-TBLR model now combines the two previous geometries and, as a result also combines the pressure levels as can be noticed in figure 13. The pressure distributions on the top and bottom faces are identical to these of C-TB model, and the left and right pressure distributions are identical to these of the C-LR model. In opposition, the drag reduction is not the sum of the DR of both the C-TB and C-LR models, but is $7.4 \%$ that is much larger! The explanation is given by the equilibrium of the pressure on all the faces due to the boat tail effect, that suppresses the three dimensional separations, and hence the longitudinal vortices as shown in figure 13(c). This result is consistent with the model proposed in [24] because it proves the role of the induced drag in the presence of the drag optimum when the wake flow is oriented on two opposite sides only. Eventually, the drag reduction obtained with the C-TBLR model is $10.7 \%$ compared to the reference squareback Ahmed model.

\section{Concluding remarks}

The control of the wake past the squareback Ahmed geometry at $\operatorname{Re}_{H}=2.510^{6}$ extends the results of the previous laboratory experiments to industrial scales. First, the flow presents a dependence on the angles of the trailing edges similar to the one depicted in [24] both in terms of trends and amplitude. The drag as a function of the top and bottom angles can be analyzed as a two dimensional contribution related to the apparent rear bluffness of the geometry and three-dimensional contributions from both the local vortices emitted at the corners of the base and the large-scale vortices associated with the lift. An optimized geometry is found presenting a 5.8\% drag reduction compared to the squareback Ahmed model. The bi-stable behavior of the wake of the reference geometry [12] is still observed in the present optimized configuration. It is characterized by a plateau of drag for very small yaw angles of sideslip, typically for $|\beta|<1.0^{\circ}$, with important fluctuations of the side force. Based on the sensitivity analysis at the laboratory scale detailed in [29], the use of a vertical disturbance at the appropriated location, i.e. near the center of the recirculation region, leads to a suppression of the bi-stable behavior and some additional drag reduction at the industrial scale. For this controlled case the drag reduction is $7.4 \%$ compared to the squareback Ahmed model. Hence 1,6\% in DR is ascribed to the presence of the control cylinder.

In parallel, an efficient method to limit the formation of the local longitudinal vortices of the optimized geometry is to orient simultaneously the flow from the sides of the geometry in order to retrieve a relative axisymmetry in the afterbody flow. In that case a drag reduction of $10.7 \%$ is obtained compared to the squareback Ahmed model. This drag reduction involves, a cavity effect bringing 3.6\% drag reduction, and the boat tail effect that together make the cumulative DR of $10.7 \%$. The cavity effect appears to be an efficient method, in our case it is coupled to a strong reduction of the side force fluctuation indicating an inhibition of the wake bi-stability. However, further experiments are needed to clarify the role of the wake bi-stability in the ability of a deep cavity to reduce the drag of the squareback Ahmed model. 


\section{Acknowledgments}

The authors wish to thank the CNRT R2A, National Centre for Technologic Research for Aerodynamics and Aeroacoustics of ground vehicles for providing the full scale wind tunnel of GIE S2A as well as the financial support for the postdoc position of A. Courbois.

[1] W. Hucho, Aerodynamics of road vehicles, SAE International, 1998.

[2] H. Choi, J. Lee, H. Park, Aerodynamics of heavy vehicles, Annual Review of Fluid Mechanics 46 (2014) $441-468$.

[3] S. Ahmed, G. Ramm, G. Faitin, Some salient features of the time-averaged ground vehicle wake, SAE Technical Paper Series 840300 .

[4] H. Lienhart, S. Becker, Flow and turbulence structure in the wake of a simplified car model, SAE Transactions 112 (6) (2003) 785-796.

[5] J. Beaudoin, O. Cadot, J. Aider, K. Gosse, P. Paranthoën, B. Hamelin, M. Tissier, D. Allano, I. Mutabazi, M. Gonzales, et al., Cavitation as a complementary tool for automotive aerodynamics, Experiments in Fluids 37 (5) (2004) 763-768.

[6] S. Krajnović, L. Davidson, Flow around a simplified car, part 1: large eddy simulation, Journal of Fluids Engineering 127 (2005) 907.

[7] S. Krajnović, L. Davidson, Flow around a simplified car. Part 2. Understanding the flow, Journal of Fluids Engineering 127 (2005) 919.

[8] E. Duell, A. George, Experimental study of a ground vehicle body unsteady near wake, SAE transactions 108 (6, part 1) (1999) 1589-1602.

[9] I. Bayraktar, D. Landman, O. Baysal, Experimental and computational investigation of Ahmed body for ground vehicle aerodynamics, SAE Technical Paper Series 2001-01-2742.

[10] S. Krajnović, L. Davidson, Numerical study of the flow around a bus-shaped body, Journal of Fluids Engineering 125 (2003) 500.

[11] M. Grandemange, M. Gohlke, O. Cadot, Reflectional symmetry breaking of the separated flow over threedimensional bluff bodies, Physical Review E 86 (2012) 035302.

[12] M. Grandemange, M. Gohlke, O. Cadot, Turbulent wake past a three-dimensional blunt body. Part 1. Global modes and bi-stability., Journal of Fluid Mechanics 722 (2013) 51-84.

[13] P. Gilliéron, A. Kourta, Aerodynamic drag reduction by vertical splitter plates, Experiments in Fluids 48 (1) (2010) $1-16$.

[14] J. Beaudoin, J. Aider, Drag and lift reduction of a three-dimensional bluff body using flaps, Experiments in Fluids 44 (4) (2008) 491-501.

[15] G. Fourrié, L. Keirsbulck, L. Labraga, P. Gilliéron, Bluff body drag reduction using a deflector, Experiments in Fluids 50 (2) (2011) 385-395.

[16] G. Pujals, S. Depardon, C. Cossu, Drag reduction of a three-dimensional bluff body using coherent streamwise streaks, Experiments in Fluids 49 (5) (2010) 1085-1094.

[17] C. Bruneau, E. Creuse, D. Depeyras, P. Gillieron, Active procedures to control the flow past the ahmed body with a $25^{\circ}$ rear window, International Journal of Aerodynamics 1 (3) (2011) 299-317.

[18] B. Khalighi, S. Zhang, C. Koromilas, S. Balkanyi, L. Bernal, G. Iaccarino, P. Moin, Experimental and computational study of unsteady wake flow behind a bluff body with a drag reduction device, SAE Technical Paper Series 2001-01-1042.

[19] C. Bruneau, E. Creusé, D. Depeyras, P. Gilliéron, I. Mortazavi, Coupling active and passive techniques to control the flow past the square back ahmed body, Computers \& Fluids 39 (10) (2010) 1875-1892.

[20] R. Littlewood, M. Passmore, Aerodynamic drag reduction of a simplified squareback vehicle using steady blowing, Experiments in Fluids 53 (2) (2012) 1-11.

[21] D. Barros, T. Ruiz, J. Borée, B. Noack, Control of three-dimensionnal blunt body wake using low and high frequency pulsed jets, International Journal of Flow Control 6 (1) (2014) 61-74.

[22] M. Rouméas, P. Gilliéron, A. Kourta, Analysis and control of the near-wake flow over a squareback geometry, Computers \& Fluids 38 (1) (2009) 60-70.

[23] R. Littlewood, M. Passmore, The optimization of roof trailing edge geometry of a simple squareback, SAE Technical Paper Series 2010-01-0510.

[24] M. Grandemange, A. Mary, M. Gohlke, O. Cadot, Effect on drag of the flow orientation at the base separation of a simplified blunt road vehicle, Experiments in fluids 54 (5) (2013) 1-10.

[25] A. Roshko, Perspectives on bluff body aerodynamics, Journal of Wind Engineering and Industrial Aerodynamics 49 (1-3) (1993) 79-100.

[26] M. Grandemange, M. Gohlke, O. Cadot, Bi-stability in the turbulent wake past parallelepiped bodies with various aspect ratios and wall effects, Physics of Fluids 25 (2013) 095103. 
[27] B. Herry, L. Keirsbulck, J. Paquet, Flow bi-stability downstream of three-dimensional double backward facing steps at zero-degree slideslip, ASME Transactions Journal of Fluids Engineering 133 (054501) (2011) 1-4.

[28] N. Lawson, K. Garry, N. Faucompret, An investigation of the flow characteristics in the bootdeck region of a scale model notchback saloon vehicle, Proceedings of the Institution of Mechanical Engineers - part D - Journal of Automobile Engineering 221 (D6) (2007) 739-754.

[29] M. Grandemange, M. Gohlke, O. Cadot, Turbulent wake past a three-dimensional blunt body. part 2. experimental sensitivity analysis., Journal of Fluid Mechanics 752 (2014) 439-461.

[30] P. Waudby-Smith, T. Bender, R. Vigneron, The GIE S2A full-scale aeroacoustic wind tunnel, SAE Technical Paper Series 2004-01-0808.

[31] M. Gohlke, J. Beaudoin, M. Amielh, F. Anselmet, Experimental analysis of flow structures and forces on a threedimensional bluff body in constant cross-wind, Experiments in Fluids 43 (4) (2007) 579-594.

[32] R. W. Kruiswyk, J. C. Dutton, Effect of a base cavity on subsonic near-wake flow, AIAA Journal 28 (11) (1990) 1885-1893.

[33] F. Browand, C. Radovich, M. Boivin, Fuel savings by means of flaps attached to the base of a trailer: field test results, SAE Technical Paper Series 2005-01-1016. 